\title{
La importancia de la colaboración entre profesorado de música de diferentes entornos: Un estudio de caso en el ámbito formal y no formal
}

\author{
Cristina Arriaga-Sanz (Universidad del País Vasco, Espanha) \\ cristina.arriaga@ehu.eus \\ Baikune De Alba-Eguíluz (Universidad del País Vasco, Espanha) \\ baikune.dealba@ehu.eus \\ Alberto Cabedo-Mas (Universitat Jaume I, Espanha) \\ cabedoa@edu.uji.es
}

\begin{abstract}
Resumen: Esta investigación analiza un proyecto cuyo objetivo fue impulsar la colaboración entre docentes de música que trabajan en tres ámbitos educativos distintos: universidad, escuelas de educación primaria y escuelas de música. Para ello se realiza una propuesta que parte de la creación musical y finaliza con la interpretación de una obra conjunta. Se plantea una forma de hacer música desde la práctica, fomentando la exploración y el gusto por hacer y expresar música. La investigación, exploratoria y descriptiva, está basada en la metodología del estudio de casos. Se analizaron entrevistas y diarios de trabajo, categorizando los resultados a partir de la propuesta de competencias profesionales de Perrenoud (2004). Los resultados subrayan la importancia de generar iniciativas y espacios colaborativos para mejorar las competencias docentes.
\end{abstract}

Palabras clave: Educación musical formal y no formal; Colaboración; Creatividad; Estudio de caso.

Resumo: Esta pesquisa analisou um projeto cujo objetivo era promover a colaboração entre professores de música que trabalhavam em três campos educacionais diferentes: universidade, escolas primárias e escolas de música. Para isso, foi feita uma proposta que começava com a criação musical e terminava com a interpretação dum trabalho conjunto. Foi proposta uma maneira de se engajar com a música a partir da prática, incentivando a exploração e o gosto na expressão e no fazer musical. A pesquisa, exploratória e descritiva, baseou-se na metodologia de estudo de caso. Foram analisadas entrevistas e diários de trabalho e os resultados foram categorizados com base na proposta de habilidades profissionais de Perrenoud (2004). Os resultados ressaltaram a importância de gerar iniciativas e espaços colaborativos para melhorar as habilidades de ensino.

Palavras-chave: Educação musical formal e não formal; Colaboração; Criatividade; Estudo de caso.

Abstract: This research analyzes a project whose objective was to promote collaboration between music teachers working in three different educational fields: University, primary schools and music schools. In order to achieve this aim a proposal is made, which starts with the musical creation and ends with the collective performance. It proposes a way of making music from practice, encouraging exploration and pleasure for making and expressing music. The exploratory and descriptive research is based on the case study methodology. Interviews and work diaries were analyzed, categorizing the results based on Perrenoud's professional competences proposal (2004). The results underscore the importance of generating initiatives and collaborative spaces to improve teaching skills.

Keywords: Formal and non-formal music education; Collaboration; Creativity; Case study.

\section{Introducción}

El informe sobre la Clasificación Internacional Normalizada de la Educación (CINE) 2011 (Organización de las Naciones Unidas para la Educación, la Ciencia y la Cultura UNESCO, 2013) señala que la educación formal es aquella educación institucionalizada e intencionada, organizada tanto por entidades públicas como por organismos privados acreditados que, en su conjunto, constituye el sistema educativo formal de un país. Las actividades que se proponen en un sistema educativo formal están específicamente diseñadas con el objetivo de la formación y el aprendizaje, y el sistema estandariza aquellos objetivos y competencias que el alumnado debe alcanzar en cada nivel (ROMI; SCHMIDA, 2009). Por su parte, la educación no formal (COOMBS, 1991; TOURIÑÁN LÓPEZ, 1996) también se define como educación institucionalizada, intencionada y organizada por un proveedor de educación. Puede representar una alternativa o complemento a la educación formal en algunos casos, y contribuye al aprendizaje a lo largo de la vida. Su estructura no implica 
necesariamente una trayectoria continua y sus destinatarios pueden pertenecer a cualquier grupo de edad. También representa en determinados contextos una forma de garantizar el acceso a una educación para todos, y a menudo aparece ligada a la comunidad, ofreciendo actividades educativas muy contextualizadas y altamente participativas (FOLKESTAD, 2006). En el informe anteriormente citado (UNESCO, 2013) se señalan elementos y aspectos coincidentes entre la educación formal y la educación no formal, como son los destinatarios, la institucionalización y la consecución de unos objetivos establecidos previamente, lo que proporciona un escenario propicio para buscar puntos de encuentro entre ambas, especialmente en el campo de la educación musical.

En España, la educación musical formal se articula fundamentalmente en las escuelas primarias y secundarias, que capacitan al estudiantado para la adquisición de capacidades perceptivas y promueve el desarrollo de las capacidades expresivas (DÍAZ; GIRÁLDEZ, 2015). Educadores e investigadores de la educación musical actual apuestan por un currículo musical en la educación obligatoria que sea equilibrado, amplio y progresivo, en el que la espontaneidad, la participación, el intercambio y la interacción de experiencias configuren un perfil pedagógico-didáctico que facilite a niños y niñas su natural capacidad creadora (DÍAZ, 2014). Al mismo tiempo, se promueve el debate entre un modelo educativo cuyo fin sea la música y una educación global a través la música (TOURIÑÁN, 2011). Además, la educación formal centrada en la capacitación de futuros profesionales de la música se articula en los conservatorios. Por su parte, la educación musical no formal recoge toda actividad educativa y sistemática que se realiza fuera del marco del sistema oficial, con la finalidad de ofrecer una formación musical a todo tipo de personas, con diversas aficiones y de cualquier edad y se atiende en las escuelas de música.

La relación entre ambas formas de aprender música ha formado parte de las investigaciones de los educadores musicales en las últimas décadas; de hecho, la educación formal y la no formal comparten intenciones pedagógicas, además de constituir sistemas organizados y sistematizados (RIAÑO, 2010). Destacamos aquí algunos ejemplos de interacción entre los dos ámbitos, como los que describen Price (2006) y Deane (2013), que subrayan la alta calidad pedagógica que pueden tener las prácticas no formales. Otra forma de colaboración es la que proponen Bamford (2011) o Heimonen (2004), entre las escuelas de música y la educación primaria. En este sentido, Díaz (2004) alude a la necesaria colaboración entre ambos ámbitos educativos y Berbel y Díaz (2014) señalan que tanto escuela primaria como escuela de música muestran predisposición a realizar acciones y proyectos colaborativos. Las autoras afirman que la realización de actividades conjuntas entre los dos tipos de instituciones, relacionando la educación musical formal y no formal, conlleva un resultado altamente positivo.

El hecho de que existan diferencias en las formas de trabajo en cada ámbito educativo supone un enriquecimiento para la educación musical, puesto que se amplía la diversidad de maneras de hacer y de aprender música. No obstante, siguiendo a Deane (2013), para abordar este enriquecimiento es fundamental buscar la manera de compartir objetivos e interrelacionarse. Coffman (2013) y Koopman (2007), entre otros, señalan que la educación musical no formal tiende de manera general a atribuir una mayor importancia a los procesos de participación en experiencias musicales, por encima de los resultados musicales finales. De este modo, los objetivos de la educación musical no formal incluyen la importancia de la interpretación grupal, de dar cabida a una amplia diversidad cultural y de estilos artísticos, y de promover el disfrute personal a través de la música. La educación musical formal, por su parte, a pesar de ha sido habitualmente planteada desde acciones que priman la excelencia musical y promueven el individualismo, comparte, por la misma 
naturaleza de la experiencia musical, la colaboración en la interpretación y el disfrute por participar en prácticas musicales. Este hecho puede constituir el punto de partida para un trabajo común. En el campo de la educación, apostar por la colaboración de profesorado de distintos ámbitos contribuye a crear experiencias positivas de aprendizaje que incrementarán la probabilidad de que el futuro docente de música construya una relación positiva con la profesión (BENNET; STANBERG, 2006). Ofrece además una oportunidad para desarrollar competencias orientadas sobrellevar las dificultades que la misma colaboración docente lleva implícitas, tales como definir criterios comunes y promover la toma de decisiones consensuada en la planificación de proyectos colaborativos.

No debemos olvidar que en las universidades españolas, a través de los estudios de grado, se forman los maestros y maestras que serán encargados de impartir la enseñanza musical en la educación primaria. Si desde la Universidad se generan experiencias que tiendan puentes entre los diferentes ámbitos formales y no formales de educación musical, obtendremos docentes con un conocimiento educativo más amplio y diverso, más comprometidos con la enseñanza de la música y más creativos. El despertar de la creatividad, definida esta como imaginación manifestada con éxito en cualquier búsqueda valiosa (ODENA, 2001), y por tanto relacionada con la noción de pensamiento imaginativo, debe ser uno de los pilares fundamentales de estas experiencias educativas. En esta línea, todos podemos ser creativos, ya que la creatividad es concebida como el desarrollo de la imaginación, de la búsqueda, y siempre conlleva el éxito, puesto que incide en el proceso y no en el producto. Se trata de desarrollar un estilo de pensamiento manifestado en acciones (ODENA, 2001), y un aprendizaje que fomente la implicación en el proceso de la persona que aprende (BEETLESTONE, 2000).

De acuerdo con Burnard y Murphy (2017, p. 38), la creatividad surge cuando el alumnado participa de forma activa en el proceso de aprendizaje, a través de la exploración, eligiendo y acordando decisiones acerca de cómo expresarse combinando diferentes sonidos y prácticas. Esta autora también incide en la importancia de que los maestros crean que son creativos y generadores de creatividad para promover el crecimiento musical, y en la necesidad de esforzarse para ayudar al alumnado a responder creativamente en su relación con la música en todo momento. En este sentido, es fundamental crear una comunidad de práctica musical, estimulando la asunción de responsabilidades, la colaboración, la autonomía, trabajar y relacionar lo conocido con lo inesperado, valorar la introducción de ideas poco habituales, la curiosidad y la toma de decisiones (BEINEKE, 2013; BURNARD; MURPHY, 2017).

Este estudio ha sido dirigido por el profesorado del departamento de Educación Musical, Plástica y Corporal de una escuela universitaria de magisterio. En él se aborda una experiencia colaborativa, activa y vivencial, entre docentes y estudiantes de escuelas de música, centros de educación primaria y el alumnado universitario de formación inicial de profesorado. Los fundamentos educativos de la experiencia tuvieron en cuenta la necesidad de acercarse a la música de forma práctica, incidiendo en el disfrute y la creación musical como motores del aprendizaje, y realizando actividades que involucraron a estudiantes y profesorado, de forma autónoma, colaborativa y comprometida para propiciar un mayor aprendizaje.

El objetivo de este estudio reside en indagar en los beneficios de la realización de actividades colaborativas en el desarrollo de competencias docentes en profesores de música de diferentes contextos educativos. 


\section{Descripción de la experiencia}

El estudio se ha llevado a cabo en una escuela de Magisterio, tres escuelas de música y cuatro centros de educación primaria de Bizkaia. Está basado en un proyecto educativo interuniversitario de creación y práctica artística, que tuvo como punto de partida la creación sonora, realizada por el alumnado universitario, de manera cooperativa, e inspirada en historias de vida anónimas (ARRIAGA; DE ALBA; TOTORICAGËNA; CUADRADO, 2017; CABEDO; RIAÑO; BERBEL, 2017).

En primer lugar, los estudiantes de Magisterio, realizaron un archivo sonoro en el que se registraron historias de vida anónimas, breves relatos de un minuto de duración. Mediante prácticas fonográficas (técnicas de grabación de audio), se creó un repositorio de experiencias vitales, recuerdos, anécdotas, etc., en el que estaban representadas todas las franjas etarias de la ciudadanía.

Posteriormente, los estudiantes formaron equipos de trabajo para abordar la fase creativa, de carácter musical. A través de una metodología cooperativa, orientada a impulsar la responsabilidad grupal, establecer grupos homogéneos con interdependencia positiva, liderazgos compartidos y un procesamiento grupal autónomo (GONZÁLEZ; GARCÍA, 2007; JOHNSON, D.; JOHNSON, R., 1989), se procedió a la escucha activa de cada historia, provocando diferentes respuestas emocionales, de acuerdo con el carácter de cada historia. Tras una puesta en común sobre las emociones emergidas, cada equipo comenzó la composición de un tema musical original para cada historia de vida, también de un minuto de duración.

Una vez que dieron por finalizada la creación e interpretación de estos primeros temas musicales para cada historia, se grabaron y fueron enviados a los participantes de las escuelas de música y las escuelas de primaria, quienes continuaron con el proceso de creación. Los estudiantes de escuelas de música añadieron ostinatos rítmicos a partir de la percusión corporal, pequeña percusión o la voz. Los estudiantes de la escuela primaria incluyeron coreografías, letras y elementos musicales destinados a enriquecer cada tema musical, tales como recursos vocales, instrumentales, o variaciones en ritmos, registros, timbres e intensidades. Las creaciones musicales finales fueron interpretadas por todos los participantes en una serie de representaciones conjuntas abiertas al público.

\section{Fases de la propuesta}

El proceso de coordinación es clave en el éxito de una acción educativa compartida (SENGE, 1992; BOLARÍN; MORENO; PORTO, 2013). En esta experiencia, la coordinación entre los diferentes docentes partió de la iniciativa del profesorado de la universidad quien, en un primer momento, estableció el contacto inicial, así como algunas de las pautas para garantizar la comunicación efectiva entre los participantes. La coordinación se organizó en cuatro fases generales en las que se llevaron a cabo las siguientes tareas:

\section{Fase 1: Toma de contacto}

Desde la universidad se estableció contacto con el profesorado de escuelas de música y de educación primaria en dos ciudades de Bizkaia con el fin de explicar las características de la experiencia, el posible desarrollo y la organización en diferentes etapas. Esta toma de contacto sirvió para acordar los grupos de estudiantes participantes en la experiencia, determinando los cursos y estableciendo un cronograma inicial de actividades. Tras un debate horizontal, se establecieron los términos de participación de cada grupo. 


\section{Fase 2: Implementación}

Durante el proceso de arreglo y composición de las ideas musicales iniciales, los docentes responsables de cada grupo ( 2 docentes de la universidad, 4 maestros de escuela primarias y 8 profesores escuelas de música) incidieron en la necesidad de mantener un diálogo regular con objeto de concretar los avances que se daban en las etapas de composición e interpretación y hacer partícipes al resto de grupos acerca de inquietudes, ideas y propuestas de los estudiantes de las escuelas de música y de los centros de educación primaria.

Una vez que el alumnado de la universidad desarrolló las ideas iniciales en las primeras composiciones, se llevó a cabo una reunión con los distintos centros con el fin de escuchar y valorar de manera conjunta las propuestas iniciales, elegir los temas a trabajar, decidir cómo gestionar las pautas compositivas a cada colectivo de estudiantes, intercambiar ideas acerca de las posibilidades compositivas e interpretativas y determinar la participación ulterior de cada grupo.

\section{Fase 3: Puesta en escena}

Con el fin de establecer las pautas para la representación final de los obras musicales resultado del trabajo conjunto se necesitó coordinar una serie de aspectos en relación a las fechas de ensayos conjuntos y de escenificación, conciliación de horarios, espacios, medios de transporte, necesidades de instrumentos y materiales, realización final del programa de concierto, etc.

\section{Fase 4: Evaluación de la experiencia}

Por último, desde la universidad se promovió una fase de evaluación de la experiencia que permitió que los docentes participantes de los centros de primaria y escuelas de música tuvieran la oportunidad de poner de manifiesto aquellas reflexiones que emergen del proceso educativo llevado a cabo. La evaluación permitió promover acciones de mejora y aprender de manera experiencial. En esta experiencia, la evaluación colaborativa resultará siempre en un proceso más rico.

En la Tabla 1 se describen algunos de los aspectos que se consideran importantes en la coordinación docente para cada una de las fases de la experiencia educativa.

Tabla 1: Aspectos clave en la coordinación docente

\begin{tabular}{|c|c|}
\hline Fases & Acciones \\
\hline 1 Toma de contacto & $\begin{array}{l}\text { - } \text { Articular las conexiones entre las competencias a desarrollar } \\
\text { - } \quad \text { Explorar posibilidades organizativas y recursos disponibles } \\
\text { - } \quad \text { Imaterminar cronogramas tentativos } \\
\text { - Tomar los acuerdos necesarios que regirán la implementación }\end{array}$ \\
\hline 2 Implementación & $\begin{array}{l}\text { - Participar de manera horizontal y dialógica en el intercambio de ideas } \\
\text { - Consensuar las tareas a realizar en cada nivel y disciplina } \\
\text { - Fomentar la práctica contextualizada y centrada en el estudiantado } \\
\text { - Adquirir responsabilidades }\end{array}$ \\
\hline 3 Puesta en escena & $\begin{array}{l}\text { - } \text { Gestionar los recursos disponibles } \\
\text { - Adaptarse a contextos diversos } \\
\text { - } \text { Asumir compromisos } \\
\text { - } \quad \text { Promorar la contribución de los demás / Empatía } \\
\text { - }\end{array}$ \\
\hline
\end{tabular}




\begin{tabular}{|l|l|}
\hline $\begin{array}{l}\text { 4 Evaluación de la } \\
\text { experiencia }\end{array}$ & - $\begin{array}{l}\text { Facilitar los canales de comunicación horizontal para la recogida de } \\
\text { datos. Entrevistas }\end{array}$ \\
& • $\begin{array}{l}\text { Posibilitar la comunicación respetuosa y sincera } \\
\text { - }\end{array}$ \\
& Debatir sobre logros y dificultades \\
\hline
\end{tabular}

\section{Método}

La finalidad de esta investigación se centra en analizar los posibles efectos positivos de la colaboración entre docentes de música de diferentes contextos educativos en la adquisición de competencias que deben dirigir la formación inicial y continua del profesorado.

Se optó por la metodología de estudio de caso (SIMONS, 2011) por diferentes razones. De acuerdo con Imbernón (2002) el hecho de describir un caso concreto y contextualizado facilita su comprensión, favorecida por la presencia de los investigadores en el campo, y su implicación facilita una descripción adecuada de la realidad que se presenta. Además, su diseño abierto y flexible potencia un proceso inductivo-deductivo necesario para la construcción del conocimiento, y su carácter interpretativo ayuda a acercarse mejor a los significados que los participantes otorgan a sus acciones y prácticas porque aportan su experiencia y comprensión del caso.

Se realizó un análisis a resultas del estudio de la experiencia educativa descrita. Se realizaron entrevistas en profundidad (KVALE, 2011) con 12 profesores de música - 4 maestros y maestras de educación primaria y 8 de escuelas de música -, y se analizaron los diarios de reflexión personal del alumnado (SICILIA-CAMACHO, 1999) de 50 estudiantes universitarios del grado de maestro. Previa a la entrevista personal con el profesorado participante, los investigadores facilitaron un guión con los temas para abordar durante la entrevista, con el fin de que el profesorado tuviera la oportunidad de conversar con su estudiantado, y poder de este modo traer las voces de estudiantes de educación primaria y escuelas de música participante en la experiencia. Esto facilitó la reflexión acerca de los aprendizajes y las limitaciones en cada grupo-clase.

Los datos recogidos fueron analizados cualitativamente, categorizando de manera deductiva la información en función de su naturaleza temática. Las categorías de análisis parten de la propuesta de Perrenoud (2004), en la que se describen grandes familias de competencias necesarias en la formación inicial y continua de la profesión docente. La Tabla 2 muestra las categorías delimitadas:

Tabla 2: Categorías del análisis

\begin{tabular}{|l|}
\hline Organizar y animar situaciones de aprendizaje \\
\hline Gestionar la progresión de los aprendizajes \\
\hline Elaborar y hacer evolucionar dispositivos de diferenciación \\
\hline Trabajar en equipo \\
\hline Participar en la gestión de la escuela \\
\hline Utilizar las nuevas tecnologías \\
\hline Afrontar los deberes y los dilemas éticos de la profesión \\
\hline Organizar la propia formación continua \\
\hline
\end{tabular}

Fuente: Perrenoud, 2004. 
La investigación tuvo en cuenta las normas éticas, garantizando la autorización de todos los participantes y la confidencialidad en la recogida, análisis y difusión de los resultados.

\section{Resultados}

\section{Organizar y animar situaciones de aprendizaje}

Organizar y animar situaciones de aprendizaje implica desarrollar otras habilidades más específicas como trabajar a partir de las representaciones del alumnado, construir y planificar secuencias de aprendizaje o comprometer a los alumnos en proyectos que, partiendo de temas que les resulten interesantes, engloban trabajos interdisciplinares o proyectos en colaboración con otros centros con el objetivo de involucrar al alumnado. En este sentido, el profesorado que ha participado en la experiencia expresa que este proyecto ha posibilitado implicar al alumnado en la construcción de su propio aprendizaje proporcionando espacios para que se exprese, entrar en diálogo con sus ideas, ayudar a superar los obstáculos e implicarles en actividades que supongan un reto al colaborar con alumnado de otros centros y participar juntos en una representación final:

Expliqué la propuesta al alumnado y escuchamos varias veces las piezas musicales que habíamos recibido. Como eran cortas, era fácil escucharlas una y otra vez. Ellos iban dando ideas y las fuimos escribiendo en la pizarra. Después elegimos las que les parecían más atractivas, pensando también que fueran viables, empezamos a probar y optamos por las que nos parecía que quedaban mejor y trabajamos sobre ellas hasta que estuvimos a gusto con el resultado (Profesor 3. Escuela de primaria)

También se señala que el trabajo en experiencias de este tipo constituye una excelente oportunidad para abrirse a nuevos contenidos, conocer otros tipos de actividades, de plantear la enseñanza/aprendizaje.

Te permite abrirte, ampliar los estilos musicales, distintos de los que trabajamos normalmente; resulta muy motivador para ellos interpretar música con niños y niñas de otras escuelas, también con el alumnado de la universidad porque había compuesto la primeras piezas, eran los autores, además mayores. Son aspectos que sin esta la colaboración no se hubiesen dado. (Profesora 8. Escuela de música)

Aunque es el último curso, es la primera vez que hemos participado en un proyecto en el que hemos podido hablar de nuestras ideas con otros compañeros. Al principio me pareció difícil; componer en grupo no es muy habitual y, además, entre nosotros hay diferencias, niveles diferentes de conocimiento. Pero al final, se ha facilitado tiempo y acompañamiento en clase para encajar las diferentes ideas y los diferentes niveles ha resultado una actividad enriquecedora (Alumna universidad. Diario de clase).

\section{Gestionar la progresión de los aprendizajes}

Algunas de las competencias que engloba este enunciado hacen referencia a la optimización del tiempo y a proponer situaciones desafiantes y ajustadas simultáneamente, para que empujen a progresar. Para ello será necesario conocer al alumnado con el que estamos trabajando. Todo el profesorado que ha participado en esta experiencia, tanto en las escuelas de música como en las escuelas de primaria conoce a los estudiantes puesto que llevan años en el mismo centro, un mínimo de 5 años y una media de 10 años, y ha 
corroborado que les pareció un reto interesante y que desde un principio se ofrecieron facilidades para ajustarse a cada uno de los niveles.

Desde el principio pensé que iba a ser una idea atractiva para ellos porque les gusta crear sus propias coreografías, trabajar con sus propias ideas. Cuando pensamos algo para representar les gusta participar en la toma de decisiones y decir qué van a hacer (Profesor 2. Escuela primaria).

Cuando escuchamos las músicas y fuimos pensando lo que íbamos a hacer, al principio estaban un poco perdidos pero poco a poco fueron saliendo ideas, yo iba animando primero a algunos porque sabía que se implican más y unos fueron tirando de otros hasta que ajustamos las ideas a las posibilidades de cada uno (Profesor 10. Escuela de música).

Por otra parte, entre el alumnado universitario, el hecho de haber acordado un calendario con las otras escuelas, de primaria y de música, aunque en ocasiones ha supuesto una cierta tensión, también les ha ayudado a gestionar las actividades que se iban haciendo y su propio progreso.

Algunas veces, teníamos dudas, al principio nos parecía muy nuevo. Pero ha habido feedback entre nosotros y el profesorado, íbamos presentando y revisando nuestro trabajo y gestionando las dificultades cada semana porque conocíamos las etapas del proyecto. A la vez, al realizar el diario de clase hemos sido también más conscientes del proceso, de nuestras necesidades, avances y dificultades. (Alumna universidad. Diario de clase).

Ampliar el alcance de las actividades propuestas a contextos más allá de la propia aula implica necesariamente desarrollar competencias orientadas a la gestión de los aprendizajes; más aún cuando existe una colaboración entre diferentes grupos de niveles educativos dispares. Para los estudiantes de las titulaciones de maestro, esta es una futura competencia profesional que de difícil modo se da en el normal desarrollo de las aulas universitarias.

\section{Elaborar y hacer evolucionar dispositivos de diferenciación}

La necesidad de adaptar mecanismos de diferenciación entre los participantes se hizo explícita a lo largo del proceso. Los mecanismos impulsados para la coordinación docente abren el espacio para aportar ideas que permitan a cada docente adaptar y contextualizar cada propuesta al contexto diferencial de su grupo-clase, sin dejar de lado una visión global y compartida de los proyectos. Se han llevado a cabo acciones en los distintos ámbitos para diversificar las prácticas docentes. Conocer, compartir y aportar ideas de manera colaborativa es, sin duda, enriquecedor para que el docente explore diferentes maneras de adaptar la diversidad académica, musical, etc., y conjugar estrategias para que esta diferenciación se produzca en el marco de una idea-proyecto amplio y comprensivo.

Un aspecto que me ha preocupado durante el proceso ha sido que algunos alumnos de la escuela primaria estudian también en la escuela de música, así que había que coordinar bien qué es lo que íbamos a hacer. Al final creo que ha resultado muy enriquecedor, porque se ha facilitado el contacto con otros colectivos y esto ayuda a conocer otras formas de trabajar y a la vez a diferenciar espacios. (Profesor 6. Escuela de música).

Trabajar con alumnado en formación y con escuelas de música a la vez te abre al mundo, conoces otras formas de trabajar, aprendes cómo aprovechar los recursos que tienes en clase, cómo incorporar a los que estudias en escuelas de música; resulta muy motivador para todos. (Profesor 2. Escuela primaria). 
A su vez, por parte del alumnado universitario, el hecho de compartir experiencias con docentes de diferentes niveles educativos y con alumnado intrínsecamente diverso en referencia a sus niveles de conocimientos y destrezas musicales permitió visibilizar la necesidad de fomentar acciones de diferenciación y adaptabilidad y conocer estrategias que docentes de diferentes colectivos emplearon.

Al principio ha sido complicado montar este trabajo porque dentro del grupo los niveles han sido muy distintos. Hemos tenido que realizar bastantes adaptaciones y nos ha costado arrancar, pero al final he quedado muy contenta con los resultados y al haber pasado bastante tiempo realizando arreglos, ha permitido también desarrollar mi creatividad. Creo que va a ser muy importante en mi futuro trabajo como profesora, porque adaptar algunas piezas musicales para que interpreten alumnos con diferentes niveles puede ser muy positivo (Alumna universidad. Diario de clase)

Creo que ha sido muy interesante poner a trabajar alumnado de diferentes niveles y formar un grupo musical para tocar y componer canciones de diferentes estilos. Yo en mi vida musical casi siempre he tocado sola y o igual con una persona, así que reunirnos con compañeros de diferentes niveles, con diferentes instrumentos, cada uno aportando desde sus conocimientos, componer y realizar arreglos para tocar canciones juntos me ha parecido que es muy enriquecedor, y puede ser muy interesante para proponer actividades en nuestro futuro profesional (Alumna universidad. Diario de clase).

Por su parte, resulta interesante impulsar la cooperación entre los alumnos y poner en práctica nuevas formas de enseñanza mutua que requieren autonomía y responsabilidad. Esto permite, por un lado, como veremos, implicar a los alumnos en su aprendizaje y en su trabajo y, por otro, promover estrategias docentes centradas en el estudiantado (MASCOLO, 2009).

\section{Trabajar en equipo}

Como señala Perrenoud (2004, p. 67-68), "la evolución de la escuela va en el sentido de la cooperación profesional [...] trabajar en grupo se convierte en una necesidad, relacionada con la evolución del oficio más que una opción personal.”

Hay distintas formas de trabajar en equipo; en esta experiencia el profesorado señala que en las ocasiones en las que han trabajado en equipo con otros centros escolares, una de las dificultades ha sido coordinar las distintas programaciones, pero que esta ocasión ha sido todo más flexible.

El hecho de trabajar con centros de música del ámbito formal y no formal nos ha ayudado como equipo a ser más flexibles, aceptar las ideas que nos llegaban y nos hemos sentido libres para hacer aportaciones, para comentarlos entre nosotros (Profesora 9. Escuela de música).

Trabajar de esta forma supone también trabajar valores, sobre todo porque se prioriza proponer frases musicales de estilos diferentes, que igual les gustan o no tanto, pero siempre hay respeto a las propuestas de los demás, porque quieren que salga bien, les resulta nuevo y están motivados (Profesora 2. Escuela de Primaria).

$\mathrm{Al}$ principio no veía claro si era posible llevar a cabo este trabajo, con alumnado de otros centros, sin conocernos. Estoy acostumbrada a trabajar en grupo con mis compañeros en la Universidad, pero fuera...Y al final siento que ha merecido la pena, cuando hemos escuchado las creaciones del alumnado de los demás centros, inspirados en nuestra ideas, ver lo que hemos podido componer entre todos, como un gran equipo,.. me ha parecido muy enriquecedor, emocionante (Alumna universidad. Diario de clase). 


\section{Participar en la gestión de la escuela}

La elaboración de proyectos es una manifestación de libertad, permite reflexionar y negociar distintas prácticas docentes y redefinir objetivos. Al mismo tiempo, si estos proyectos son colaborativos, promueven la necesidad de gestionar de manera efectiva los recursos necesarios, compartir recursos y esfuerzos, negociar la participación en diferentes modelos de gestión, asumir compromisos o centrar energías en la promoción de actitudes positivas en torno a la resolución de problemas. Una colaboración efectiva puede posibilitar que el compartir la gestión de recursos en diferentes contextos suponga una ampliación de los recursos disponibles.

Administrar los recursos de la escuela también posibilita un ejercicio autónomo de la profesión, permite elegir y tomar decisiones colectivamente, pero entre las cualidades necesarias del profesorado es fundamental fomentar la participación activa del alumnado dentro de la gestión de las actividades del aula.

Ha sido especialmente motivadora para nuestros alumnos la libertad para elegir los recursos con los que íbamos a componer la obra. Además, hemos trabajado estilos musicales que no solemos trabajar y han utilizado recursos y materiales poco habituales. El hecho de realizar un concierto final con la propuesta, en un escenario, con alumnado que no conocen, de diferentes edades, ha favorecido la implicación (Profesor 10. Escuela de música).

También me ha parecido interesante que es la primera vez que participamos en la gestión de una sala externa al centro para hacer una representación, con un técnico de sonido. (Profesor 2. Escuela primaria)

Al principio estaba preocupada porque pensaba que no teníamos recursos como grupo para hacer una buena composición, pero a medida que avanzaba el tiempo me he dado cuenta de que tenemos muchos recursos en el aula con los que podemos hacer buena música y de los que no somos conscientes (Alumna universidad. Diario de clase).

\section{Utilizar las nuevas tecnologías}

No es nuevo que las tecnologías de la información y la comunicación han modificado la forma de comunicarse, de trabajar, decidir y pensar del profesorado (PERRENOUD, 2004). De acuerdo con Giráldez (2013), las Tecnologías de Información y Comunicación (TIC), constituyen uno de los elementos claves en el proceso, de redefinición de la educación, siempre que a través de las mismas se potencie la creatividad y la innovación, situándolas en un contexto y definiendo previamente cuál es su función en la educación y qué acciones deben llevarse a cabo para favorecer su integración en el aula. En este caso, se ha potenciado la utilización de software específico de composición edición y práctica musical a través de actividades innovadoras, fomentando la colaboración en la creación e interpretación musical conjunta, aspecto que ha sido especialmente valorado por el alumnado universitario.

Hoy día la utilización de la tecnología es fundamental en el proceso de aprendizaje de música... Hemos aprendido que las TIC no son el objetivo mismo sino que el objetivo es utilizar las TIC para aprender; además de la importancia de desarrollar la competencia digital como futuros docentes es fundamental una utilización crítica y creativa de las TIC (Alumna universidad. Diario de clase). 


\section{Afrontar los deberes y los dilemas éticos de la profesión}

La participación colaborativa en esta experiencia permite explorar una visión de la educación musical preocupada por la formación integral de la persona. Un modelo educativo que contemple una educación a través de la música (TOURIÑÁN LÓPEZ; LONGUEIRA, 2010). Igualmente, se ha incidido en el análisis de la relación pedagógica, la autoridad y la comunicación en clase, negociando las normas con el alumnado, en base a valores democráticos, haciendo todo lo posible para definir las reglas y tomar decisiones colectivas.

Fomenta actitudes positivas y seriedad al subir a un escenario y escuchar los trabajos que han hecho los demás es siempre enriquecedor (Profesor 1. Escuela de primaria).

Supone trabajar los valores, el respeto a otros estilos de música, el respeto al turno de los demás (Profesor 3. Escuela de música).

Participar en esta actividad me ha permitido sentirme orgullosa y contenta del trabajo realizado. (Alumna universidad. Diario de clase).

Nos ha ayudado a trabajar las relaciones en la educación desde la cercanía, que nos puede ayudar a construir una sociedad más justa, más feliz, y nos ha puesto en contacto con un modelo de aprendizaje servicio, que en mi opinión es una forma de trabajar muy interesante, porque al recoger pequeñas historias para componer música sobre ellas nos hemos abierto a la comunidad. Esto es muy importante en nuestra profesión (Alumna universidad. Diario de clase).

\section{Organizar la propia formación continua}

La formación continua de los profesores está directamente relacionada con una práctica guiada por la reflexión, ya que cuando el profesorado es capaz de analizar sus prácticas puede aprovechar mejor y sacar más partido de las nuevas modalidades de formación continua (PERRENOUD, 2004). En este sentido, el hecho de negociar un proyecto con compañeros ayuda a provocar replanteamientos que conducirán a un progreso colectivo. La realización de talleres y cursos resulta ser poco práctica para transferir lo aprendido en la práctica cotidiana del aula y resulta más efectiva una revisión de las relaciones entre el profesorado, la comunicación, y se enriquece con las aportaciones de otros docentes, estudiantes y distintos agentes de la educación a través del diálogo, el intercambio de ideas y facilitando la reflexión y la discusión (ARRIAGA, 2014; BAUTISTA et al., 2016; COBO; VAAMONDE; HERRERO; CABEDO, 2016).

He comprobado que se puede aprender mucho a través del proceso de exploración y de comunicación. Me gustaría profundizar en este proceso en mi futuro profesional. Ofrecería un espacio a los alumnos para explorar individualmente y luego componer piezas musicales en grupo y organizaría espacios para compartirlo (Alumna universidad. Diario de clase).

\section{Conclusiones}

El proyecto llevado a cabo ha posibilitado implicar al alumnado universitario de formación inicial del profesorado en la construcción y en la gestión de su propio aprendizaje, ha permitido estructurar las diferentes prácticas musicales y acciones docentes que se dan en los distintos ámbitos y conjugar estrategias para que esta diferenciación se produzca en el marco de una idea-proyecto amplio y comprensivo. Esta forma de trabajar en el ámbito escolar afecta a las estructuras organizativas y metodológicas y organiza los contenidos desde la perspectiva globalizadora (TRUJILLO SÁEZ, 2017). Esto se pone de manifiesto 
no sólo en la organización de los módulos de aprendizaje universitarios, sino en todo el conjunto de la escuela primaria y la escuela de música participante.

Los docentes participantes coinciden que han experimentado la riqueza de conocer distintas formas de trabajar relacionadas con la creatividad y la tecnología, de aprovechar los recursos que tienen, integrando al alumnado de diferentes edades y niveles. Se ha reflexionado sobre el rol docente, avanzando hacia funciones menos directivas, en las que se da voz al alumnado, además del enriquecimiento que supone trabajar con profesorado de distintos ámbitos para avanzar en una mayor coordinación. La práctica reflexiva en el ejercicio docente (SCHÖN, 1992) es un proceso clave para la mejora de la calidad educativa de nuestros centros; una colaboración docente bien articulada ofrece nuevas posibilidades para ampliar el conocimiento sobre la acción educativa, promover la reflexión en y durante la acción, y estructurar una fase necesaria posterior en la que se posibilite la evaluación y la reflexión sobre la acción educativa. Las posibilidades de la colaboración docente como parte íntegra del desarrollo profesional docente son al mismo tiempo altamente valoradas por los mismos docentes (BAUTISTA et al., 2016).

La participación en esta experiencia ha hecho posible explorar una visión de la educación musical implicada en la formación integral de la persona (TOURIÑÁN LÓPEZ; LONGUEIRA, 2010), fomentando la colaboración en la creación e interpretación musical conjunta y el hecho de ofrecer la posibilidad de preparar juntos una interpretación final ha permitido crear una práctica musical auténtica, participativa, más significativa. Este modelo coincide con estructuras educativas que apuestan por un aprendizaje que hace uno de la música como una praxis social (REGELSKI, 2009), en el que se apuesta por la promoción experiencias musicales participativas, frente a un paradigma educativo tradicional, en el que se ha optado por acciones musicales presentacionales (TURINO, 2008).

La flexibilidad entre los agentes educativos facilita la coordinación de distintas programaciones $\mathrm{y}$, principalmente, fomentada una práctica guiada por la reflexión, reconociendo la diversificación en el aprendizaje pero estableciendo metas y principios educativos comunes dialogados. Es por tanto coherente con paradigmas educativos que, en primer lugar, apuestan por un enfoque de enseñanza que se centra en la naturaleza del compromiso y la participación en comunidades reflexivas y, en segundo lugar, que fomenta el tipo de autorreflexión y autoconocimiento necesarios para que las personas puedan sostener y desarrollar sus identidades propias. Este aspecto es clave para desarrollar competencias orientadas a la creación de comunidades educativas democráticas y flexibles (QUICKE, 1997).

Todo lo expuesto refuerza además el interés de generar iniciativas y espacios colaborativos donde la creatividad es el punto de partida, ya que contribuyen a reforzar la relación de los estudiantes con la música, manteniendo el interés por continuar con este tipo de actividades prácticas, lo que resulta beneficioso para el alumnado y para los centros de enseñanza. Se puede concluir que el modelo estudiado ha generado un interesante referente para el profesorado y alumnado implicado, y ha servido de ayuda para mejorar las competencias docentes, aspecto que constituye en objetivo principal de esta experiencia.

La experiencia que se ha descrito ha implicado un gran esfuerzo de compromiso tanto por parte del alumnado como del profesorado; además de trabajo en colaboración, hay una alta participación activa de todos y todas para proporcionar un aprendizaje de calidad. Todo ello nos ha permitido extraer algunas implicaciones especialmente relevantes para la práctica educativa: 
a. El trabajo colaborativo entre docentes es un ingrediente significativo para poder desarrollar metodologías activas y participativas con éxito en la educación musical. La colaboración es una oportunidad de aprendizaje que posibilita la mejora de la tarea docente. El resultado positivo del trabajo realizado sugiere la relevancia de poner en marcha experiencias de innovación práctica en el aula de formación inicial y continua de docentes de música.

b. Es necesario aplicar en las aulas estrategias metodológicas que ofrecen posibilidades para ser aplicadas por los futuros profesores y profesoras. Tener contacto con experiencias reales ayuda al alumnado a conectar teoría y práctica. El alumnado considera de gran valor contar con la oportunidad de compartir, participar de primera mano con profesionales de centros educativos y creen que ello les ayuda a ampliar su perspectiva sobre la educación musical y lo que como profesionales pueden llevar a cabo. Por ello creemos que es necesario orientar acciones de formación inicial y continua de docentes que tomen en consideración la colaboración en diferentes ámbitos educativos.

c. Las experiencias basadas en Aprendizaje Basado en Proyectos (ABP) en educación musical están fundamentalmente articuladas en propuestas en las que un único grupo-clase organiza un proyecto, pero en raras ocasiones se incluyen diferentes niveles de aprendizaje; más aún cuando hablamos de niveles de edades tan dispares y entornos formales y no formales. La gestión de colectivos diversos requiere de la adquisición de competencias que son claves para el proceso educativo. Es responsabilidad de las instituciones de formación de docentes ofrecer espacios para posibilitar nuevas formas de aprendizaje y colaboración. Por ello creemos que es necesario orientar acciones de formación inicial y continua de docentes que tomen en consideración la colaboración en diferentes ámbitos educativos.

\section{Referencias}

ARRIAGA, Cristina. Seguir aprendiendo: la formación continua. In: GIRÁLDEZ, Andrea. (Coord.). Didáctica de la música en primaria. Madrid: Síntesis, 2014. p. 147-160.

ARRIAGA, Cristina; DE ALBA, Baikune; TOTORICAGÜENA, Maricel; CUADRADO, Francisco. Historias que emocionan: grabación, creación y edición sonora como experiencia colaborativa en la Universidad. Dedica. Revista de Educação e Humanidades. Granada (España), n. 12, p. 131153, 2017.

BAMFORD, Anne. Arts and cultural education in Norway 2010-2011. Bodø (Noruega): University of Nordland. Nasjonalt senter for kunst og kultur I oplæringen, 2011. Recuperado de < http:// www.kulturskoleradet.no/upload/bruker/dokumenter/Dokumentarkiv/10_Forskning/2012_ Arts_and_Cultural_Education_in_Norway.pdf $>$.

BAUTISTA, Alfredo; TOH, Guo-Zheng; WONG, Joanne. Primary school music teachers' professional development motivations, needs, and preferences: Does specialization make a difference? Musicae Scientiae, Hannover (Alemania), nov. 2016. doi: 10.1177/1029864916678654

BEETLESTONE, Florence. Niños creativos, enseñanza imaginativa. Madrid: La Muralla, 2000. $184 \mathrm{p}$.

BEINEKE, Viviane. Creative learning and communities of practice: Perspectives for music education in the school. International Journal of Community Music, Bristol (UK), v. 6, n. 3, p. 281-290, 2013.

BENNET, Dawn; STANBERG, Andrea. Musicians as teachers: developing a positive view through collaborative learning partnerships. International Journal of Music Education, Needlands (Australia), v. 24, n. 3, p. 219-230, 2006. 
BERBEL, Noemy; DÍAZ, Maravillas. Educación formal y no formal: un punto de encuentro en la educación musical. Aula abierta, Oviedo, v. 42, n. 1, p. 47-52, 2014.

BOLARÍN, María José Martínez; MORENO, María Ángeles Yus; PORTO, Mónica Currás. Coordinación docente e interdisciplinariedad: análisis de su contribución a la adquisición de competencias docentes y discentes. Revista de Docencia Universitaria. REDU, Valencia, v. 11, n. 2, p. 443-462, 2013. Recuperado el 4 de abril de 2017 en <http://red-u.net>.

BURNARD, Pamela; MURPHY, Regina. Enseñar música de forma creativa. Madrid: Ediciones Morata, 2017. 282 p.

CABEDO, Alberto; RIAÑO, María Elena; BERBEL, Noemy. UniTICarte: Redes Colaborativas para el desarrollo de proyectos creativos en la formación de Maestros en la Universidad. Dedica. Revista de Educação e Humanidades, Granada (España), n. 12, p. 119-129, 2017

COBO, Joana; VAAMONDE, Romary; HERRERO, Sofía; CABEDO, Alberto. Reflexiones en torno a las percepciones de docentes de música en su actividad profesional: cuestiones acerca de organización curricular y tiempo. Materials: Quaderns de Didàctica Artística, Mallorca, v. 4, p. 44-52, 2016.

COFFMAN, Don. Common ground for community music and music education. International Journal of Community Music, Bristol (UK), v. 6, n. 3, p. 273-280, 2013.

COOMBS, Philip H. (Coord.). Estrategia para mejorar la calidad de la educación superior en México. México: SEP-FCE, 1991. 104 p.

DEANE, Kathryn. Community music and music pedagogy: Collaborations, intersections and new perspectives: A personal reflection. International Journal of Community Music, Bristol (UK), v. 6, n. 3, p. 291-294, 2013.

DÍAZ, Maravillas. La música en la educación primaria y en las escuelas de música: la necesaria coordinación. Revista Electrónica Complutense de Investigación en Educación Musical, Madrid, v. 1, n. 2, 2004. Recuperado de <http://pendientedemigracion.ucm.es/info/reciem/v1n2.pdf>.

. Enseñar música en el siglo XXI. In: GIRÁLDEZ, Andrea. (Coord.). Didáctica de la música en primaria. Madrid: Síntesis, 2014. p. 13-30.

DÍAZ, Maravillas; GIRÁLDEZ, Andrea. Music Education in Spain: Beyond the Curriculum. In: FIGUEIREDO, Sergio; SOARES, José; FINK SCHAMBECK, Regina (Ed.). The Preparation of Music Teachers: A Global Perspective. Porto Alegre: ANPPOM, 2015. p. 259-280.

FOLKESTAD, Göran. Formal and informal learning situations or practices vs formal and informal ways of learning. British Journal of Music Education, Cambridge (UK), v. 23, n. 2, p. 135-145, 2006. doi: 10.1017/S0265051706006887

GIRÁLDEZ, Andrea. Enseñar artes en un mundo digital: diez propuestas para la formación del profesorado. Pós, Belo Horizonte, v. 3, n. 5, p. 85-104, 2013.

GONZÁLEZ, Natalia; GARCÍA, María Rosa. El Aprendizaje Cooperativo como estrategia de Enseñanza-Aprendizaje en Psicopedagogía (UC): repercusiones y valoraciones de los estudiantes. Revista Iberoamericana de Educación, Madrid, v. 42, n. 6, p. 1-13, 2007.

HEIMONEN, Marja. Music and Arts Schools. Extra-curricular Music Education: A Comparative Study. Action, Criticism and Theory for Music Education, Buffalo (NY, USA), v. 3, n. 2, 2004. Recuperado de <http://act.maydaygroup.org/articles/Heimonen3_2.pdf >.

IMBERNÓN, Francisco. La investigación educativa como herramienta de formación del profesorado. Reflexión y experiencias de investigación educativa. Barcelona: Graó, 2002. 185 p. 
JOHNSON, David W.; JOHNSON, Robert T. Cooperative Learning: What Special Education Teachers Needs to Know". The Pointer (en la actualidad: Preventing School Failure: Alternative Education for Children and Youth), Abingdon (UK), v. 33, n. 2, p. 5-10, 1989.

KOOPMAN, Constantijn. Community music as music education: on the educational potential of community music. International Journal of Music Education, Needlands (Australia), v. 25, n. 2, 151-163, 2007. doi: 10.1177/0255761407079951

KVALE, Steinar. Las entrevistas en investigación cualitativa. Madrid: Ediciones Morata, 2011. $200 \mathrm{p}$.

MASCOLO, Michael F. Beyond Student-Centered and Teacher-Centered Pedagogy: Teaching and Learning as Guided Participation. Pedagogy and the Human Sciences, North Androver (MA, USA), v. 1, n. 1, p. 3-27, 2009.

ODENA, Oscar. Developing a framework for the study of teachers' views of creativity in music education, Goldsmiths Journal of Education, London, v. 4, n. 1, p. 59-67, 2001.

PERRENOUD, Philippe. Diez nuevas competencias para enseñar. Barcelona: Graó, 2004. 168 p.

PRICE, David. Personalising Music Learning. London: Paul Hamlyn Foundation, 2006. Recuperado de < http://webarchive.nationalarchives.gov.uk/20130401151715/http://www.education.gov.uk/ publications/eOrderingDownload/0478-2006PDF-EN-02.pdf $>$.

REGELSKI, Thomas A. Curriculum Reform: Reclaiming "Music" as Social Praxis. Action, Criticism, and Theory for Music Education, Buffalo (NY, USA), v. 8, n. 1, p. 66-84, 2009.

ROMI, Shlomo; SCHMIDA, Mirjam. Non-formal education: a major educational force in the postmodern era. Cambridge Journal of Education, Cambridge (UK), v. 39, n. 2, p. 257-273, 2009. doi: 10.1080/03057640902904472

RIAÑO GALÁN, María Elena. Educación musical formal y no formal en el G9. Eufonía: Didáctica de la música, Barcelona, v. 48, p. 87-98, 2010.

SENGE, Peter M. La Quinta Disciplina. Barcelona: Granica, S. A. Sánchez Santamaría, 1992. $490 \mathrm{p}$.

SIMONS, Helen. El estudio de caso: Teoría y práctica. Madrid: Ediciones Morata, 2011. 264 p.

SICILIA-CAMACHO, Álvaro. El diario personal del alumnado como técnica de investigación en Educación Física. Apunts: Educación física y deportes, Barcelona, v. 58, p. 25-35, 1999.

SCHÖN, Donald A. La formación de profesionales reflexivos: Hacia un nuevo diseño de la enseñanza y el aprendizaje en las profesiones. Barcelona: Ediciones Paidós, 1992. 320 p.

TOURIÑÁN LÓPEZ, José Manuel; LONGUEIRA MATOS, Silvana. La música como ámbito de educación. Educación «por» la música y educación «para» la música. Teoría de la educación, Salamanca, v. 22, n. 2, p. 151-181, 2010.

TOURIÑÁN LÓPEZ, José Manuel. Análisis conceptual de los procesos educativos formales, no formales e informales. Teoría de la educación, Salamanca, v. 8, p. 55-80, 1996.

. Claves para aproximarse a la educación artística en el sistema educativo: educación "por” las artes y educación "para” un arte. ESE: Estudios sobre educación, Pamplona, v. 21, p. 61-81, 2011.

TURINO, Thomas. Music as Social Life: The Politics of Participation. Chicago: University of Chicago Press, 2008. 280 p. 
TRUJILLO SÁEZ, Fernando. Aprendizaje basado en proyectos: Líneas de avance para una innovación centenaria. Textos. Didáctica de la Lengua y de la Literatura, Barcelona, v. 78, p. 15-22, 2017.

UNESCO. Clasificación Internacional Normalizada de la Educación, CINE 2011. Montreal: Instituto de Estadística de la UNESCO, 2013. Recuperado de <http://www.uis.unesco.org/ Education/Documents/isced-2011-sp.pdf $>$.

QUICKE, John. Reflexivity, community and education for the learning society. Curriculum Studies (en la actualidad: Pedagogy, Culture \& Society), Abingdon (UK), v. 5, n. 2, p. 139-161, 1997. doi: 10.1080/14681369700200009

Cristina Arriaga Sanz es profesora titular del área de Didáctica de la Expresión Musical en la Facultad de Educación de Bilbao (Universidad del País Vasco). Doctora en Filosofía y Ciencias de la Educación por esta misma universidad y titulada en Pedagogía Musical. Es miembro del consejo de dirección de la Revista de Psicodidáctica y del consejo editorial de la revista Eufonía: Didáctica de la Música. Ha realizado trabajos y publicaciones acerca de la motivación para el aprendizaje de música en primaria, principalmente en su relación con las formas de actuación del profesorado y las interacciones que se producen durante la práctica educativa musical. También ha investigado acerca de la música tradicional y su aplicación didáctica, el uso de los recursos multimedia en la formación artística del alumnado de Magisterio y los valores y la educación musical.

\begin{tabular}{l} 
Alberto Cabedo Mas es profesor titular de música en la Universitat Jaume I de Castellón, España. Estudió música, \\
en la especialidad de violín, y obtuvo un Máster en Música en la Academia Estonia de Música y Teatro, en Tallinn, \\
Estonia y, posteriormente, un Máster en Estudios de Paz, Conflictos y Desarrollo en la Universitat Jaume I. Es doctor \\
por esta misma Universidad. Es autor de diversas publicaciones en foros de difusión y revistas científicas nacionales \\
e internacionales. Es co-director de la revista Eufonía: Didáctica de la Música, y miembro del comité editorial de \\
diversas revistas científicas. Sus intereses de investigación incluyen la educación musical, el patrimonio musical, la \\
convivencia, la interculturalidad y la transmisión musical a través de las culturas. \\
\hline
\end{tabular}

Baikune De Alba Eguiluz es profesora adjunta en el Departamento de Didáctica de la Expresión Musical, Plástica y Corporal, en la Universidad del País Vasco, España. Doctora por esta misma universidad y Titulada Superior en Música en las especialidades de Composición y de Dirección de Orquesta. Ha realizado asimismo los cursos de postgrado de Master en Administración Pública (Universidad del País Vasco) y Master en Dirección y Gestión de Centros Educativos (Universidad de Deusto/Universidad Autónoma de Barcelona). Sus trabajos de investigación más recientes se centran en la educación musical en sus diferentes ámbitos, en la innovación educativa y en la formación del profesorado. 